\title{
A NOTE ON KLOOSTERMAN SUMS
}

\section{ALBERT LEON WHITEMAN}

1. Introduction. In recent years the Kloosterman sum

$$
A_{k}(n)=\sum_{h \bmod k}^{\prime} \exp (2 \pi i n(h+\bar{h}) / k)
$$

has played an increasingly important role in the analytic theory of numbers. The dash ' beside the summation symbol indicates that the letter of summation runs only through a reduced residue system with respect to the modulus. The number $\bar{h}$ is defined as any solution of the congruence $h \bar{h} \equiv 1(\bmod k)$, and $n$ denotes an arbitrary integer. It was shown by Salié ${ }^{1}$ almost fifteen years ago that $A_{k}(n)$ may be evaluated explicitly when $k$ is a power of a prime. Salié's result is given by the following theorem.

THEOREM. Let $k=p^{\alpha}, \alpha \geqq 2,(n, k)=1$, where $p$ denotes an odd prime. Then,

(i) if $\alpha$ is even,

$$
A_{k}(n)=2 k^{1 / 2} \cos (4 \pi n / k)
$$

(ii) if $\alpha$ is odd,

$$
A_{k}(n)=\left\{\begin{aligned}
2(n \mid k) k^{1 / 2} \cos (4 \pi n / k) & \text { for } p \equiv 1(\bmod 4) \\
-2(n \mid k) k^{1 / 2} \sin (4 \pi n / k) & \text { for } p \equiv 3(\bmod 4)
\end{aligned}\right.
$$

The symbol $(n \mid k)$ denotes, as is usual, the Legendre symbol.

Salié's proof of his theorem is based upon induction. In the present note a direct proof is given. The method consists in introducing a transformation which expresses the Kloosterman sum in terms of Gauss sums and certain types of Ramanujan sums.

2. Two lemmas. A Gauss sum may be defined by

$$
G_{h, k}=\sum_{m=0}^{k-1} \exp \left(2 \pi i h m^{2} / k\right)
$$

We shall find it convenient to write $G$ instead of $G_{1, k}$. The following lemma ${ }^{2}$ is classical.

Received by the editors October 26, 1944.

${ }^{1}$ Hans Salié, Über die Kloostermanschen Summen $S(u, v ; q)$, Math. Zeit. vol. 34 (1931) pp. 91-109.

2 See, for example, Edmund Landau, Vorlesungen iber Zahlentheorie, vol. 1, p. 153. 
LEMma 1. If $k$ is an odd integer and $(h, k)=1$, then

$$
G_{h, k}=(h \mid k) G
$$

and

$$
G=i^{((k-1) / 2)^{2}} k^{1 / 2} .
$$

We shall also need the following lemma.

Lemma 2. Let $p$ denote an odd prime; let $n$ and $\alpha$ denote positive integers. Then

$$
\sum_{h \bmod p^{\alpha}}^{\prime} \exp \left(2 \pi i n h / p^{\alpha}\right)=\left\{\begin{array}{c}
p^{\alpha}-p^{\alpha-1} \text { if } p^{\alpha} \mid n, \\
-p^{\alpha-1} \text { if } p^{\alpha} \nmid n \text { but } p^{\alpha-1} \mid n, \\
0 \text { if } p^{\alpha-1} \nmid n(\alpha>1) .
\end{array}\right.
$$

Furthermore, if $\alpha$ is odd, and if we put $n_{1}=n / p^{\alpha-1}$ when $p^{\alpha-1} \mid n$, we have

$$
\sum_{h \bmod p^{\alpha}}^{\prime}\left(h \mid p^{\alpha}\right) \exp \left(2 \pi i n h / p^{\alpha}\right)=\left\{\begin{array}{l}
0 \text { if } p^{\alpha} \mid n, \\
i^{((p-1) / 2)^{2}}\left(n_{1} \mid p\right) p^{\alpha-1 / 2} \\
\text { if } p^{\alpha-1} \mid n \text { but } p^{\alpha} \nmid n, \\
0 \text { if } p^{\alpha-1} \nmid n(\alpha>1) .
\end{array}\right.
$$

The first part of this lemma follows at once from a well known transformation formula ${ }^{3}$ for Ramanujan sums or may easily be proved directly. The second part of the lemma may be established in the following way:

If $p^{\alpha} \mid n$, then

$$
\sum_{h \bmod p^{\alpha}}^{\prime}\left(h \mid p^{\alpha}\right) \exp \left(2 \pi i n h / p^{\alpha}\right)=\sum_{h \bmod p^{\alpha}}^{\prime}\left(h \mid p^{\alpha}\right)=0 .
$$

If $p^{\alpha}\left\{n\right.$ but $p^{\alpha-1} \mid n$, then by (1)

$$
\begin{aligned}
\sum_{h \bmod p^{\alpha}}^{\prime}\left(h \mid p^{\alpha}\right) \exp \left(2 \pi i n h / p^{\alpha}\right) & =\sum_{h \bmod p^{\alpha}}^{\prime}(h \mid p) \exp \left(2 \pi i n_{1} h / p\right) \\
& =\left(n_{1} \mid p\right) p^{\alpha-1} \sum_{h=1}^{p-1}(h \mid p) \exp (2 \pi i h / p) .
\end{aligned}
$$

But it is easy to show that ${ }^{4}$

$$
G_{1, p}=\sum_{h=1}^{p-1}(h \mid p) \exp (2 \pi i h / p) .
$$

Hence, by (2), the lemma is established in this case. Finally, if $p^{\alpha-1} \nmid n$,

${ }^{3}$ See, for example, Landau, loc. cit., vol. 1, bottom of p. 280.

4 See, for example, Landau, loc. cit., vol. 1, p. 155. 


$$
\begin{aligned}
\sum_{h \bmod p^{\alpha}}^{\prime}\left(h \mid p^{\alpha}\right) \exp \left(2 \pi i n h / p^{\alpha}\right) & \\
= & \sum_{h \bmod p^{\alpha}}^{\prime}\left(h+p \mid p^{\alpha}\right) \exp \left(2 \pi i n(h+p) / p^{\alpha}\right) \\
= & \exp \left(2 \pi i n / p^{\alpha-1}\right) \sum_{h \bmod p^{\alpha}}^{\prime}\left(h \mid p^{\alpha}\right) \exp \left(2 \pi i n h / p^{\alpha}\right)=0,
\end{aligned}
$$

where we have noted that $\exp \left(2 \pi i n / p^{\alpha-1}\right) \neq 1$ since $p^{\alpha-1}\{n$. This completes the proof of Lemma 2.

3. Proof of Salié's theorem. Let us first observe that (2) may be written in the form $1=(-1 \mid k) G^{2} / k$. Using (1) we may now transform the Kloosterman sum $A_{k}(n)$ in the following manner.

$$
\begin{aligned}
A_{k}(n)= & (-1 \mid k) G^{2} / k \sum_{h \bmod k}^{\prime} \exp \left(2 \pi i\left(-n^{2} h-\bar{h}\right) / k\right) \\
= & (-1 \mid k) G / k \sum_{h \bmod k}^{\prime} \exp \left(2 \pi i\left(-n^{2} h-\bar{h}\right) / k\right) \\
& \cdot \sum_{m=0}^{k-1}(h \mid k) \exp \left(2 \pi i h m^{2} / k\right) \\
= & (-1 \mid k) G / k \sum_{h \bmod k}^{\prime} \sum_{m=0}^{k-1}(h \mid k) \exp \left(2 \pi i h\left(m^{2}-n^{2}-\bar{h}^{2}\right) / k\right) \\
= & (-1 \mid k) G / k \sum_{h \bmod k}^{\prime} \sum_{m=0}^{k-1}(h \mid k) \exp \left(2 \pi i h\left(m^{2}-n^{2}+2 m \bar{h}\right) / k\right)
\end{aligned}
$$

since $m+\bar{h}$ runs through a complete residue system with respect to the modulus $k$ whenever $m$ does. Interchanging signs of summation we get

$$
\begin{aligned}
A_{k}(n)=(-1 \mid k) G / & k \sum_{m=0}^{k-1} \exp (4 \pi i m / k) \\
& \cdot \sum_{h \bmod k}^{\prime}(h \mid k) \exp \left(2 \pi i\left(m^{2}-n^{2}\right) h / k .\right.
\end{aligned}
$$

At this point we divide the discussion into two cases according as $\alpha$ is even or odd. For $\alpha$ even, we have

$$
A_{k}(n)=G / p^{\alpha} \sum_{m=0}^{p^{\alpha}-1} \exp \left(4 \pi i m / p^{\alpha}\right) \sum_{h \bmod p^{\alpha}}^{\prime} \exp \left(2 \pi i\left(m^{2}-n^{2}\right) h / p^{\alpha}\right) .
$$

Referring to (3) we see that the last sum equals zero except when $p^{\alpha-1} \mid m^{2}-n^{2}$. Now the solutions ${ }^{5}$ of the congruence $m^{2} \equiv n^{2}\left(\bmod p^{\alpha}\right)$

'See, for example, G. H. Hardy and E. M. Wright, An introduction to the theory of numbers, pp. 95-96. 
are all given by $m \equiv \pm n\left(\bmod p^{\alpha}\right)$, and the solutions of the congruence $m^{2} \equiv n^{2}\left(\bmod p^{\alpha-1}\right), m \bmod p^{\alpha}$, where $m^{2} \not n^{2}\left(\bmod p^{\alpha}\right)$, are $m \equiv \pm n+q p^{\alpha-1}\left(\bmod p^{\alpha}\right), 1 \leqq q \leqq p-1$. Hence, applying the first part of Lemma 2, we obtain

$$
\begin{aligned}
& A_{k}(n)=G / p^{\alpha}\left\{\left(p^{\alpha}-p^{\alpha-1}\right) \exp \left(4 \pi i n / p^{\alpha}\right)\right. \\
& \left.+\left(p^{\alpha}-p^{\alpha-1}\right) \exp \left(-4 \pi i n / p^{\alpha}\right)-p^{\alpha-1} \sum_{\alpha=1}^{p-1} \exp \left(4 \pi i\left( \pm n+q p^{\alpha-1}\right) / p^{\alpha}\right)\right\} \\
& \quad=2 G \cos (4 \pi n / k),
\end{aligned}
$$

which completes the proof of the theorem in the case in which $\alpha$ is even.

We next consider the case which arises when $\alpha$ is odd. For this purpose we return to (6) and obtain

$$
\begin{aligned}
A_{k}(n)=\left(-1 \mid p^{\alpha}\right) G_{1, p^{\alpha}} / p^{\alpha} \sum_{m=0}^{p^{\alpha}-1} \exp \left(4 \pi i m / p^{\alpha}\right) & \sum_{h \bmod p^{\alpha}}^{\prime}\left(h \mid p^{\alpha}\right) \\
& \exp \left(2 \pi i\left(m^{2}-n^{2}\right) h / p^{\alpha}\right) .
\end{aligned}
$$

From (4) we see that the last sum is zero except when $p^{\alpha-1} \mid m^{2}-n^{2}$ but $p^{\alpha} \nmid m^{2}-n^{2}$. Furthermore, let us observe that the number $n_{1}$, defined in Lemma 2 , is here of the form $\pm 2 n q+q^{2} p^{\alpha-1}$. Hence, proceeding as we did in the case in which $\alpha$ is even, we get

$$
\begin{aligned}
A_{k}(n)= & \left(-1 \mid p^{\alpha}\right) G / p^{\alpha} \sum_{\alpha=1}^{p-1} \exp \left(4 \pi i\left( \pm n+q p^{\alpha-1}\right) / p^{\alpha}\right) \\
& \cdot\left\{i^{((p-1) / 2)^{2}}( \pm 2 n q \mid p) p^{\alpha-1 / 2}\right\} \\
= & \left(-1 \mid p^{\alpha}\right) G_{1, p^{\alpha}} / p^{\alpha}\left\{\left(n \mid p^{\alpha}\right) G_{1, p} p^{\alpha-1}\right. \\
& \cdot \exp \left(4 \pi i n / p^{\alpha}\right) \sum_{q=1}^{p-1}(2 q \mid p) \exp (4 \pi i q / p) \\
& \left.+\left(-n \mid p^{\alpha}\right) G_{1, p} p^{\alpha-1} \exp \left(-4 \pi i n / p^{\alpha}\right) \sum_{q=1}^{p-1}(2 q \mid p) \exp (4 \pi i q / p)\right\} \\
= & \left(n \mid p^{\alpha}\right) G_{1, p^{\alpha}} / p^{\alpha}\left\{\left(-1 \mid p^{\alpha}\right) G_{1, p}^{2} p^{\alpha-1} \exp \left(4 \pi i n / p^{\alpha}\right)\right. \\
& \left.+G_{1, p}^{2} p^{\alpha-1} \exp \left(-4 \pi i n / p^{\alpha}\right)\right\} .
\end{aligned}
$$

This completes the proof of the theorem in this case in view of Lemma 1.

4. Concluding remarks. The reader may have wondered why the case $\alpha=1$ is excluded in Salié's theorem. The reason is that Salié's 
method breaks down in this case as, indeed, does ours. For the sake of completeness we shall now show that when $\alpha=1$ our method leads merely to a transformation formula.

For $k=p$, the last sum in (6) becomes a Gauss sum in view of (5). Thus we have by (1) and (2)

$$
\begin{aligned}
A_{p}(n) & =(-1 \mid p) G / p \sum_{m=0}^{p-1} \exp (4 \pi i m / p) \sum_{h=1}^{p-1}(h \mid p) \exp \left(2 \pi i\left(m^{2}-n^{2}\right) h / p\right) \\
& =(-1 \mid p) G^{2} / p \sum_{m=0}^{p-1}\left(m^{2}-n^{2} \mid p\right) \exp (4 \pi i m / p) \\
& =\sum_{m=0}^{p-1}\left(m^{2}-4 n^{2} \mid p\right) \exp (2 \pi i m / p) .
\end{aligned}
$$

Hence, we obtain the transformation formula

$$
\sum_{h=1}^{p-1} \exp (2 \pi i n(h+\bar{h}) / p)=\sum_{m=0}^{p-1}\left(m^{2}-4 n^{2} \mid p\right) \exp (2 \pi i m / p),
$$

which may, of course, be established directly without much difficulty.

Various sums related to the Kloosterman sum $A_{k}(n)$ have been evaluated by Salié ${ }^{6}$ and Lehmer. ${ }^{7}$ The author has verified that the method of this paper may be employed to obtain new derivations of these results.

Washington, D. C.

- Loc. cit.

7 D. H. Lehmer, On the series for the partition function, Trans. Amer. Math. Soc. vol. 43 (1938) pp. 271-295. 